\title{
Effect of Bias Load on Positioning Characteristics of Shape Memory Alloy Actuator Based on Resistance Feedback Control
}

\author{
Yuji TAKEDA*, Takaei YAMAMOTO** and Toshio SAKUMA** \\ *TAKE R\&D, Shizuoka 426-0087, E-mail: crsth527@ybb.ne.jp \\ **Faculty of Engineering, Oita University, Oita 870-1192
}

The actuators using shape memory alloy can work as an actuator to control or retain positioning without using sensor device. In order to utilize the shape memory effect, the bias force such as coil spring and constant load is required to deform the shape memory alloy in martensitic phase. In this work, the effect of a bias load on the positioning characteristics by resistance feedback control is investigated. The produced actuator is heated by electric current and it can control the position using a constant mass and a bias spring as a bias load. The Ti-Ni-Cu alloy wire is used as an actuator element. The positioning characteristics such as settling velocity, positional error, and position accuracy are discussed in relation to the bias load. The results show that the settling velocity at heating decreases and the settling velocity at cooling increases with increasing in the bias load. Furthermore, the positional error at heating is very small and the positioning control against the desired position during heating can be controlled accurately.

Key words: actuator, position control, resistance feedback control, shape memory alloy, bias mechanism

\section{INTRODUCTION}

The actuators using shape memory alloy (SMA) can work as an actuator to control or retain positioning without using sensor device [1], because SMA is such a superior element that the element itself has a sensing function in it. This type of sensor-less system brings about many merits like simplification of the system and easy and simple operability. SMA has a characteristic that electric resistance varies due to the phase transformation in the process of heating and cooling [2], which occurs as a conversion of strain and force. The actuators, applying this characteristic to position control of SMA with resistance feedback, have been reported [3].

However, the resistance values of SMA show non-linear characteristics with hysteresis, resulting in giving different position information for rising and lowering processes of temperature [2]. This means that the real-time resistance values can not be fed back, as is, for the positioning information. Some attempts have been carried out to realize a position control system using the PID control by resistance feedback [4-8], but they remain only at the continuous positioning control or power control. Thus, most applications are limited for the back and forth movement between two given points (not the intermediate points in between). If positioning can be achieved for an arbitrary position, the applications of such actuators will be wide spread.

We have proposed the new resistance feedback control system by using the method of setting OFF time and Blanking time and have showed that positioning can be set and retained at an arbitrary position [3].

In this work, a position control system with two kinds of biasing mechanism is produced and evaluated.
Investigation is made on such positioning characteristics as settling velocity at heating/cooling and position accuracy in relation to the bias load. Furthermore, the effect of biasing mechanism that is a constant mass and a bias spring on positioning characteristics is investigated.

\section{EXPERIMENTAL PROCEDURE}

\subsection{Position control system}

The produced position control system by resistance feedback control with two kinds of biasing mechanism is shown in Fig.1. The SMA element used for the actuator is $\mathrm{Ti}-\mathrm{Ni}-\mathrm{Cu}$ alloy, which has a relatively small transformation temperature hysteresis. The alloy wire, which is memory processed in a linear shape, has a length of $400 \mathrm{~mm}$ and a diameter of $0.156 \mathrm{~mm}$. The transformation temperatures obtained from the resistance-temperature characteristics are $A f=350 \mathrm{~K}$, $\mathrm{As}=312 \mathrm{~K}, \mathrm{Ms}=336 \mathrm{~K}$ and $\mathrm{Mf}=290 \mathrm{~K}$. A constant mass is 40-130 gr.

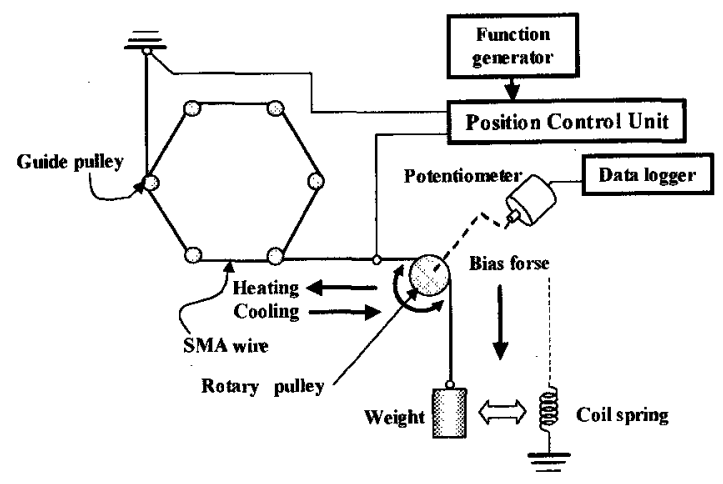

Fig.1 A schematic diagram of position control system with two kinds of biasing mechanism. 
The system is composed of pulleys, a constant mass/a bias spring, a potentiometer, and a controller circuit. The SMA wire with an overall length of $400 \mathrm{~mm}$ is wound 2 rounds around the 6 guide pulleys. The potentiometer is linked to the rotation axis of a rotary pulley so that the positioning data for the resistance feedback control can be measured.

In the potentiometer feedback system, the voltage variation of the potentiometer and the preset input voltage are fed into a differential amplifier as the input, thus the potentiometer as a position sensor enables a servo loop with a simple circuitry.

Since SMA is a non-linear and non-reversible element with hysteresis characteristics, it can generate a shape recoverable strain through conduction heating but this strain can not be brought back to the non-strain position with conduction of electricity. Therefore, in order to position-control the SMA, electricity needs to be conducted till the resistance value of SMA reaches a preset value and then be put off so that position does not over-pass the desired position. The important point for the accurate positioning is how to control the repetition of "on" time (heating process) and "off" time (cooling

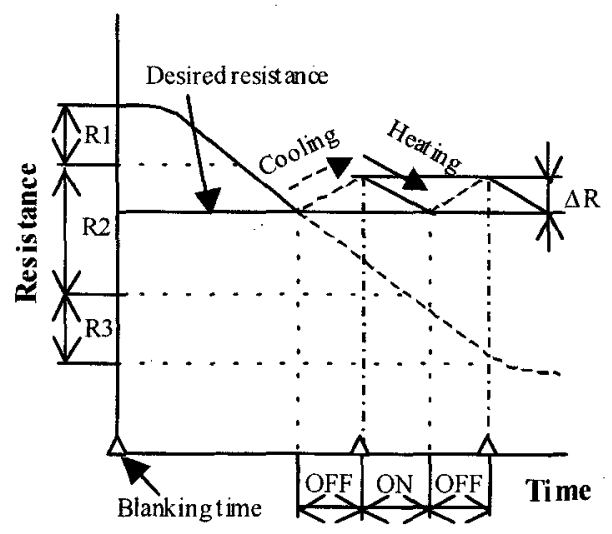

Fig. 2 Control method of conduction-off time.

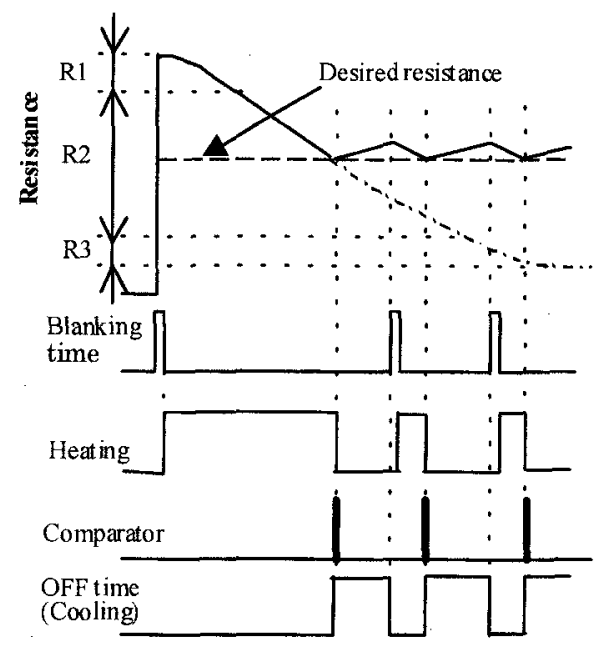

Fig.3 Time chart of comparator. process). The SMA shows non-linear hysteresis characteristics, lowering its resistance value with temperature rise (i.e. negative resistance characteristics). Taking this into account, conduction-off time is controlled, in the process of resistance lowering, with three different regions of the resistance values $R 1, R 2$, and R3 as shown in Fig.2. The three-comparator circuits selectively switch these R1, R2, and R3 regions, and since resistance characteristics show negative resistance, it is so arranged that the comparators do not detect the resistance values at the time of conduction starting (i.e. blanking time). Driver is a constant current type to detect the real-time resistance. In Fig.2, depending on the region ( $R 1, R 2$, or $R 3$ ) where the position input (i.e. resistance value) is, the resistance feedback control is achieved by switching the OFF time through the cycle of "end of off-time", "conduction on", "detection by comparator" and "conduction off". The times shown in $\Delta$ mark in the figure are blanking times, and these blanking times are essential to the resistance feedback loop for the comparator to identify the resistance at phase transformation. Figure 3 shows the time chart of comparator switching, that depend on which region (R1, R2, or R3) the resistance value of ordered input is.

\subsection{Measurement of dead time and settling time}

Fig. 4 shows the variation of the input voltage and the displacement with time. The $\mathrm{P} 1$ and the $\mathrm{P} 2$ denote the initial controlled position and the desired position, respectively. The dead time $t_{\mathrm{DH}}$ at heating is defined as the time when the displacement does not generate during heating. The dead time $t_{D C}$ at cooling is also defined as the time when the displacement does not generate during cooling.

After the lapse of $t_{\mathrm{DH}}$, the time from the initial controlled position $\mathrm{P} 1$ to the desired position $\mathrm{P} 2$ by heating is defined as the settling time $t_{\mathrm{SH}}$. The settling time $t_{\mathrm{Sc}}$ at cooling is also defined as the time from the desired position $\mathrm{P} 2$ to the initial controlled position $\mathrm{P} 1$ after the lapse of $t_{D C}$.

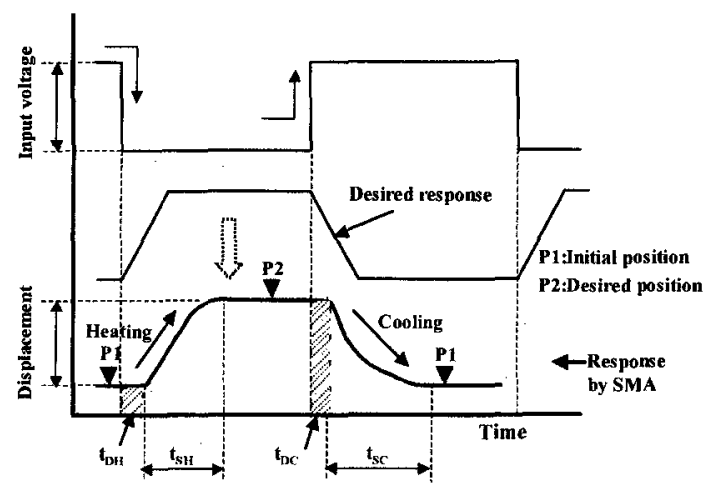

Fig.4 Measurement of dead time and settling time. 
2.3 Measurement of the positional error and the accuracy of positioning

Fig. 5 shows the measurement method of the positional error and the accuracy of positioning. The positional error is obtained by the displacement difference $\Delta \mathrm{P} 1$ between the initial position $\mathrm{P} 1$ and the controlled position $\mathrm{P} 1$ after cooling.

The accuracy of position control is defined by the fluctuations of desired position during heating and the accuracy of positioning is estimated by the displacement difference $\Delta \mathrm{P} 2$ between the desired position $\mathrm{P} 2$ and the controlled position $\mathrm{P} 2$.

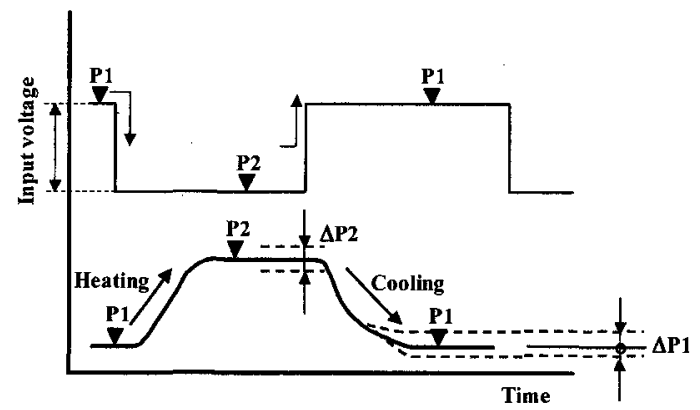

Fig.5 Measurement of hysteresis and accuracy of positioning.

\section{RESULTS AND DISCUSSION}

\subsection{Variation of settling speed with bias load}

Fig. 6 shows the effect of bias load on the settling speed $V_{\mathrm{SH}}$ at heating as a function of a constant mass and a bias spring. The load of bias spring is corresponding to that of deforming to the desired position $\mathrm{P} 2$. The $\mathrm{V}_{\mathrm{SH}}$ is obtained by following equation.

$$
\mathrm{V}_{\mathrm{SH}}=(\mathrm{P} 2-\mathrm{P} 1) / \mathrm{t}_{\mathrm{SH}}[\mathrm{mm} / \mathrm{s}]
$$

It is well known that in stress-temperature curve, the austenite start and finish temperatures As and Af increase linearly with increasing in stress. When the bias load become large, it is necessary for recovering the SMA wire to the original length to heat it up to high temperature. Therefore, the settling time $t_{\mathrm{SH}}$ becomes

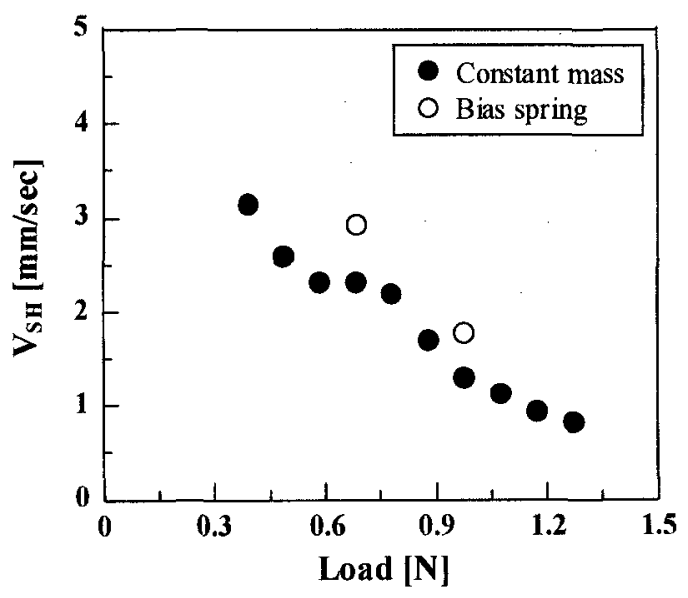

Fig.6 Variation of $V_{\text {SH }}$ with bias load at heating. large according to the increase of bias load and the settling speed $\mathrm{V}_{\mathrm{SH}}$ at heating decreases with increasing in bias load as shown in Fig.6. In case of bias spring, the settling speed $\mathrm{V}_{\mathrm{SH}}$ also decreases with increasing in bias load. Furthermore, since the load of bias spring becomes small according to the shape recovery of SMA, the settling speeds are faster than those of a constant mass.

On the contrary, when the position moves to the controlled position P1 by cooling from the desired position P2, the settling time is dependent on the cooling speed of SMA wire. Since the martensite start temperature Ms also increases with increasing in stress, the cooling time when the temperature of SMA wire is below Ms becomes short according to the increase of stress.

Fig. 7 shows the effect of bias load on the settling speed $V_{\mathrm{SC}}$ at cooling as a function of a constant mass and a bias spring. The $\mathrm{V}_{\mathrm{SC}}$ is obtained by following equation.

$$
\mathrm{V}_{\mathrm{SC}}=(\mathrm{P} 2-\mathrm{P} 1) / \mathrm{t}_{\mathrm{sc}}[\mathrm{mm} / \mathrm{s}]
$$

In case of a constant mass, since the cooling time decreases with the increasing in bias load, the settling speed $V_{S C}$ increases with increasing in bias load. In case of bias spring, since the Ms decreases according to the decrease of the load of spring, the $V_{S C}$ is slower than that of a constant mass.

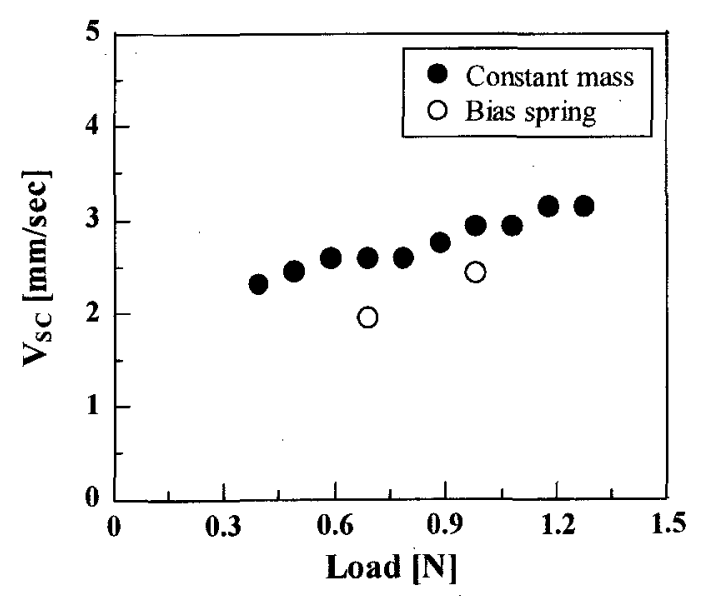

Fig.7 Variation of $\mathrm{V}_{\mathrm{SC}}$ with bias load at cooling.

\subsection{Variation of positional error with bias load}

Fig. 8 shows the variation of positional error $\Delta \mathrm{P} 2 / \mathrm{P} 2$ at heating with bias load. The amount of fluctuations of two different biasing mechanisms is almost $0.06 \%$ against the desired position $\mathrm{P} 2$ and is independent on the bias load.

On the contrary, the positional error at cooling varies with bias load. Fig. 9 shows the variation of positional error $\Delta \mathrm{P} 1 / \mathrm{P} 1$ at cooling with bias load. The amount of fluctuations of biasing mechanism of a constant mass is almost $0.06 \%$ below about $1.1 \mathrm{~N}$ and is same as that of heating. However, when the bias load of a constant mass 


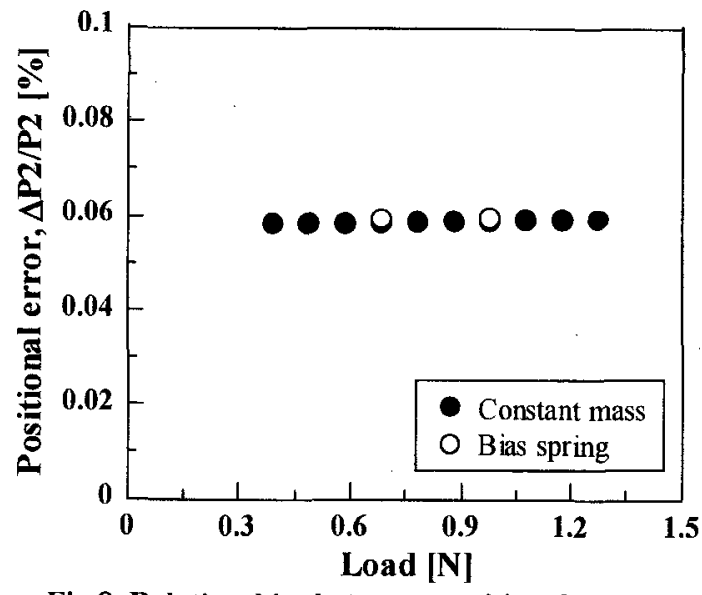

Fig.8 Relationship between positional error $\triangle \mathrm{P} 2 / \mathrm{P2}$ and bias load at heating.

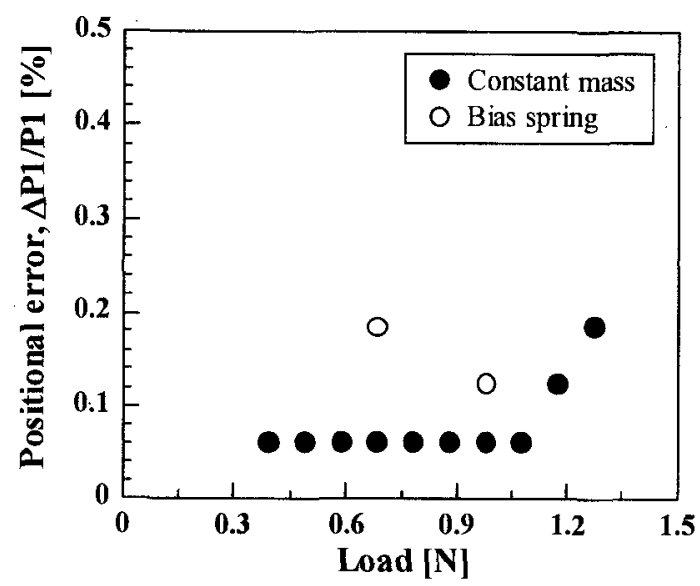

Fig.9 Relationship between positional error $\Delta \mathrm{P} 1 / \mathrm{Pl}$ and bias load at cooling.

is above about $1.2 \mathrm{~N}$ and the biasing mechanism is bias spring, the positional errors become larger. This reason is considered to be due to the uncontrollable cooling speed.

\subsection{Accuracy of position control}

Fig. 10 shows the variation of the accuracy of position control with time as a function of two different biasing mechanisms of a constant mass and a bias spring. In the figure, Emax is defined by $\Delta \mathrm{P} 2 \max / \mathrm{P} 2$, where $\Delta \mathrm{P} 2 \max$ is the maximum fluctuation value during the control of desired position P2. Emax is $0.04-0.72 \%$ and the positioning control in both biasing mechanisms can be controlled accurately.

\section{CONCLUSION}

A position control system with two different biasing mechanisms is produced and evaluated. Investigation is made on such positioning characteristics as the settling velocity and the positional error at heating/cooling, and the position accuracy in relation to the bias load. The following conclusions are derived from results.

(1) Since the austenite start and finish temperatures increase linearly with increasing in stress, the settling

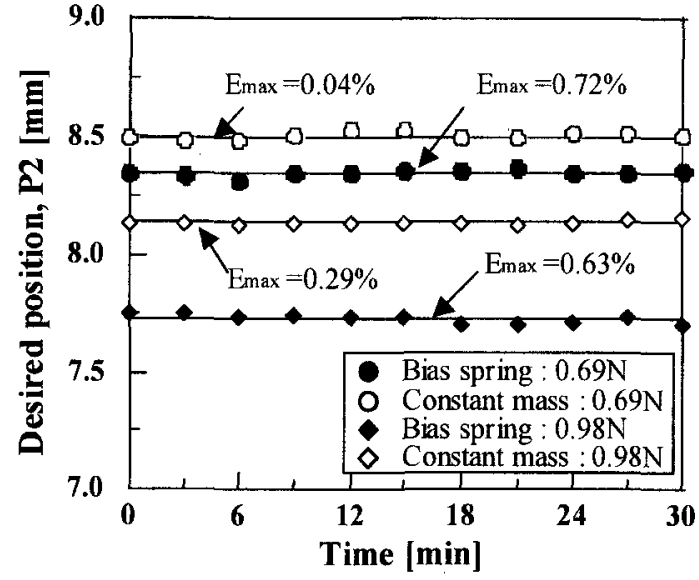

Fig.10 Variation of positional error against desired position $P 2$ with time.

time at heating increases with increasing in the bias load. Therefore, the settling velocity decreases with increasing in the bias load.

(2) On the other hand, the settling velocity at cooling increases with increasing in the bias load because the martensite start temperature increases according to the increase of the bias load

(3) In case of a bias spring, since its bias load varies with the displacement, the settling velocity is larger than that of a constant mass in the process of heating and is smaller than that in the process of cooling.

(4) The positional error at heating is very small in both biasing mechanisms. However, since the cooling speed can not be controlled, the positional error at cooling increases in the case of bias spring and in the higher bias load range in the case of constant mass.

(5) The positional error hardly varies with time and the positioning control in both biasing mechanisms can be made accurately.

\section{REFERENCES}

[1] D. Homma, Journal of the Robotics Society of Japan, 8-4, 107-109 (1990).

[2] Y. Takeda and T. Sakuma, Trans. MRSJ, 31-2, 295-298(2006).

[3] S. Hirose, K. Ikuta and Y. Umetani, Journal of the Robotics Society of Japan, 4-2, 15-24(1985).

[4] K. Kuribayashi, System \& Control, 27-9, 49-57 (1983).

[5] K. Kojima, T. Hasegawa and S. Majima, Trans. Jpn. Soc. Mech. Eng., C, 65-640, 120-125(1999).

[6] S. Hirose, K. Ikuta and S. Tsukamoto, Journal of the Robotics Society of Japan, 5-2, 3-16(1987).

[7] K. Ikuta, Journal of the Robotics Society of Japan, 8-4, 113-115(1990).

[8] H. Sagawa, M. Hashimoto and K. Sato, Journal of the Robotics Society of Japan, 6-2, 21-28(1987).

(Received January 20, 2007;Accepted May 10, 2007) 